\title{
Mechanical Modeling of Al-Mg Alloy Open-Cell Foams
}

\author{
Kwan Moo Ryu ${ }^{1}$, Jae Young $\mathrm{An}^{1}$, Won-Seung Cho ${ }^{1}$, Yeon-Chul Yoo ${ }^{1}$ and Hyoung Seop Kim²,* \\ ${ }^{1}$ Department of Materials Science and Engineering, Inha University, Incheon, 402-751, Korea \\ ${ }^{2}$ Department of Metallurgical Engineering, Chungnam National University, Daejeon, 305-764, Korea
}

\begin{abstract}
A finite element analysis was employed to simulate the deformation behavior of Al-Mg alloy foams of 92\% porosity during compressive testing and the simulated results were compared with experimental ones. The crushable foam model developed in ABAQUS software for compressible foam materials could well reproduce the uniaxial compressive behavior in terms of stress-strain response and deformed geometry. In particular, the effect of friction between the dies and the foam specimen was discussed.
\end{abstract}

(Received November 24, 2004; Accepted January 25, 2005)

Keywords: metallic foam, aluminum-magnesium alloy, mechanical properties, plastic deformation behavior, finite element analysis, friction effect

\section{Introduction}

Metallic foams ${ }^{1-3)}$ having a cellular or porous structure made up of an interconnected network of strut or plates are currently attracting a great deal of attention from the materials research community due to their superior combination of high strength-to-weight ratio, high heat resistance and good sound and energy absorption capacity. They are extensively used in a variety of applications not only in structural parts such as automotive industry, aerospace industry, ship building, railway industry, building industry, machine construction, sporting equipment and biomedical industry but also in functional parts. ${ }^{4,5)}$

There have been extensive reports ${ }^{1-3,6)}$ on the production and mechanical properties of metallic foams, most of which are the relationships among processing conditions, structures and properties. Indeed, the properties of foam materials are determined by a number of geometric and structural properties, among which the relative density is the most important for mechanical properties. In attempts to understand the mechanical response of foam materials under loading, a number of micromechanical models have been developed. Most of the proposed mechanical models are employed by analytical $^{1,7-9)}$ (typically e.g. Gibson and Ashby ${ }^{10)}$ ) or numerical $^{10)}$ approaches, e.g. the finite element method (FEM), based on idealized representations of the repetitive cubic or hexagonal arrays of the cell structure. However, it is recognized that most metallic foams show little periodicity. ${ }^{6)}$ Although most of these theoretical models and their analytical solutions can describe the stress-strain behavior related to cell materials and structures and other phenomena, it is felt that we still need to investigate the local deformation states of the testing specimens. Indeed, even if the deformation behavior looks homogeneous according to the measured stress-strain curves, local deformation within the sample can be heterogeneous because there are many nonlinear factors which induce local stress concentration, such as sample and die geometries, friction between sample and die surfaces, etc. In order to investigate the local deformation states in the samples, numerical analyses in conjunction with adequate

*corresponding author, E-mail: hskim@cnu.ac.kr constitutive models are necessary. FEM incorporating adequate yield models or constitutive equations, has been successfully applied to analyzing mechanical behavior not only of conventional solid materials but also of porous materials. ${ }^{11,12)}$ Since calculating the deformation of the all cell walls of engineering parts composed of metallic foams without periodic conditions is practically impossible under the current computing power, it is necessary to try computationally efficient methods in an engineering sense. Hence, some attempts using FEM have been made to simulate deformation behavior of metallic foams by assuming the cell structure as compressible continuum, although it is difficult to quantify the precise significance of the stochastic nature of the cell and cell wall geometry in terms of its effect on the mechanical performance. ${ }^{6}$ Indeed, the continuum-based FEM of compressible materials has been successfully applied to crash $^{13)}$ and indenting ${ }^{6,14)}$ situations of metallic foams.

On the other hand, Al-based alloy foams offer a great potential for the improvement of crash behavior of automobile components due to their low density, specific properties for energy absorption and low producing cost relative to other lightweight foams. In particular, the aluminum-magnesium (Al-Mg) alloys are widely used in car industry and ship building, replacing steel and fiber reinforced plastic parts due to their excellent properties such as high strength, corrosion resistance and weldability by applying various heat treatment processes. ${ }^{15,16)}$ In this paper we investigate the deformation behavior of Al-Mg alloy foams under compression by using experiments and a numerical approach based on compressible continuum material model of FEM. Especially, the effect of friction between the dies and the foam specimen is investigated, which is an important factor controlling deformation homogeneity but has not been studied well up to now as far as the authors know.

\section{Finite Element Analysis and Experimental Methods}

Al-Mg alloy foams, having nominal composition of $\mathrm{Mg}$ 2.3, Si-0.24, Cu-0.02, Zn-0.002 mass\%, were prepared using the DUOCEL process, the casting method molded by polymer preforms, ${ }^{17)}$ by ERG, Oakland, CA. The foam samples were prepared in the form of $20 \times 20 \times 30 \mathrm{~mm}^{3}$ 

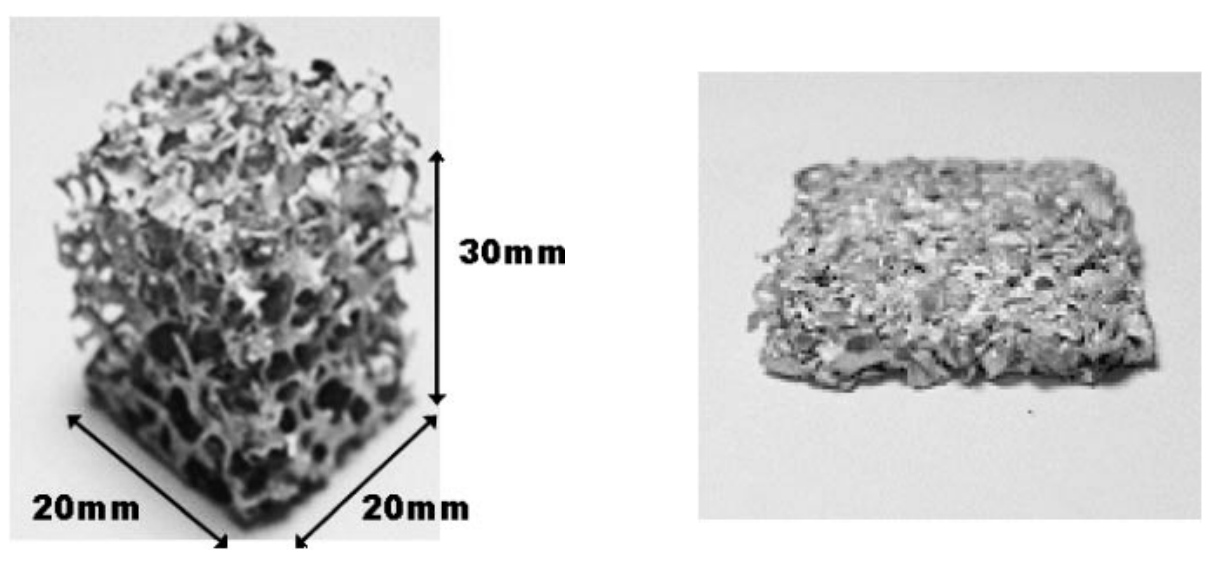

Fig. 1 Typical pictures of (a) initial and (b) $90 \%$ compressed Al-Mg alloy foams.

cuboid, see Fig. 1. All samples were subjected to the apparent density measurements by weighting using analytical balance and accurate dimension measurements, resulting in density of $0.22 \mathrm{~g} / \mathrm{cm}^{3}$ and relative density of 0.08 . Typical cell size was $3.5 \mathrm{~mm}$. For the solid Al-Mg alloy of the same composition, tensile strength was $275 \mathrm{MPa}, 0.2 \%$ offset yield stress was $152 \mathrm{MPa}$ and elongation was about $10 \%$.

The Al-Mg foam specimens were inserted between parallel platens without any lubrication. Uniaxial compression tests were carried out on a servo-hydraulic testing machine equipped with a $10 \mathrm{kN}$ load cell. All tests were conducted under displacement control with a cross head speed of $0.3 \mathrm{~mm} / \mathrm{s}$ which corresponds to an initial strain rate of $0.01 \mathrm{~s}^{-1}$.

Uniaxial compressive tests were simulated using the three dimensional elasto-plastic FEM code ABAQUS/ STANDARD $6.4 .^{18)}$ In fact, although the analytical solution under the assumption of uniaxial and homogenous deformation gives a lot of useful global average information, it gives little information on the local inhomogeneous tri-axial stress state behavior. Hence, FEM based on the macroscale continuum mechanics is essential in order to obtain the local deformation behavior, especially in analyzing the engineering parts.

Due to the symmetric conditions of geometry, boundary and loading, 1/8 of the specimen was taken as a calculating domain, see Fig. 2. An eight node brick element with reduced integral (C3D8R) was used and the number of the element was 1500. A contact pair was defined between the ram and the foam part. Contact surface interaction was modeled by specifying a Coulomb friction coefficient $\mu$. In order to investigate the effect of the friction, it is meaningful to consider the minimum and maximum friction states. For this purpose, the two extreme frictional cases, i.e. $\mu=0$ of sliding condition and sticking condition, were considered. Real friction conditions exist between the two limit cases.

Since the metallic foams are compressible, contrary to non-porous metallic materials, pressure dependence of plastic yielding needs to be implemented in constitutive equation. Various pressure dependent constitutive models have been proposed ${ }^{19,20)}$ and used for simulating densification behavior of porous and powder materials. ${ }^{21,22)}$ For a present constitutive material model, the crushable foam

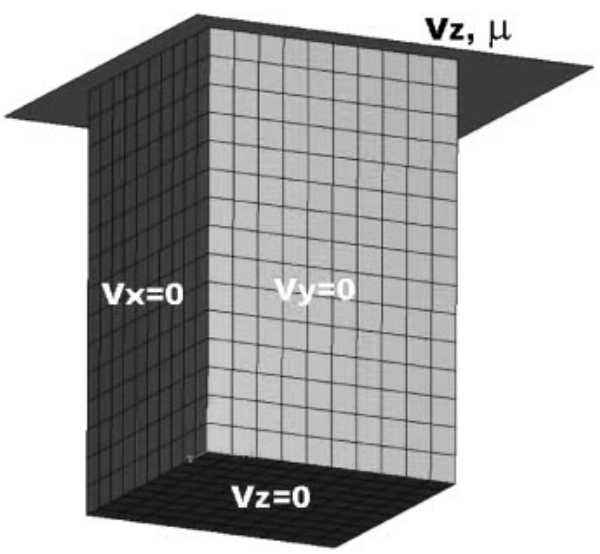

Fig. 2 Initial mesh and pressing die with boundary, friction and loading conditions.

plasticity model ${ }^{13)}$ available in ABAQUS was used. The material input data, i.e. volumetric hardening represented by a multiple stress-strain table for the Al-Mg foam in the crushable foam model was obtained from the best-fitted curves under the same strain rate $\left(0.01 \mathrm{~s}^{-1}\right)$ condition. Anisotropy was ignored based on the previous experimental results that the relative orientation of force and forming directions is of minor importance. ${ }^{23)}$ The strain rate dependence was neglected, because little strain rate sensitivity was measured, ${ }^{9)}$ although there is a report that the plateau stress of the $\mathrm{Al}$ foam exhibited strong strain rate sensitivity at a dynamic strain rate. ${ }^{24)}$

The calculation was performed on a HP690 supercomputer. The run time for one simulation up to $90 \%$ reduction in height was less than a few minutes.

\section{Results and Discussion}

Figure 3 shows the calculated and experimental stressstrain curves during the compression testing of the Al-Mg foam of $8 \%$ relative density. The predicted stress-strain behavior by the sliding friction condition of FEM is in very good agreement with experimental values. It should be noted that the good agreement of the simulated and experimental curves results from the usage of the material data obtained 


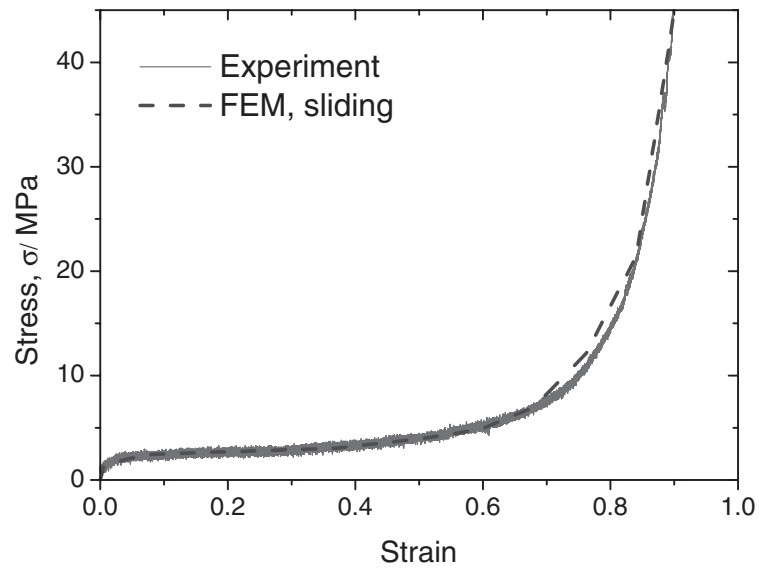

Fig. 3 Calculated and experimental stress vs. strain curves during uniaxial compression testing of the Al-Mg alloy foam.

from the experimental results. Nevertheless, this good agreement confirms the validity of the crushable foam model and the usefulness of the current finite element modeling associated with the compressible model for the real forming and deformation applications. The curves show typical stress vs. strain behavior for cellular materials having three distinct stages. ${ }^{1)}$ At stage I, under relatively low loads up to about 0.01 of strain, the material is linear elastic. At stage II, under some critical level of load, the cell begins to collapse by elastic buckling, plastic yielding or brittle fracture, depending on the nature of the cell wall properties. Cell collapse then progresses at a roughly constant load, producing a roughly horizontal stress plateau, until relatively large strain (typically about 0.7 ). At stage III, opposing cell walls begin to meet and touch. The stress then rise steeply as the material densifies, and finally the material attains bulk-like properties. It is known that the extended plateau is particularly important for the foam application as an energy absorber. To absorb the impact energy effectively, a material is required to exhibit an extended stress plateau.

Figure 4 shows the initial and calculated deformed geometries at various states in (a) sliding and (b) sticking friction conditions. In the frictionless (sliding friction) compression, Fig. 4(a), there is little lateral expansion even until the reduction in height reaches $80 \%$ of the initial height, resulting in plastic Poisson ratio's of small value but not zero exactly. Since there is a very small relative lateral motion of the workpiece, we can expect that deformation will not be affected much by friction behavior. In case of the sticking frictional condition, Fig. 4(b), compressive deformation is more localized near the die contact region than the central region. Due to the effect of friction between the workpiece and the die surfaces, the movement of the surface region of the workpiece was retarded (even if it is small) and barreling occurred. From the deformation after $80 \%$ of reduction in height, deformation becomes localized in the corners and the deformed geometry is very nonuniform, see Fig. 4(b). Comparing the deformed geometries to the experimental ones, e.g. Fig. 1(b), it can be seen that the real situation resides between the two extreme (sliding and sticking) conditions, as can be expected.

Volume contraction of the sample during compressive

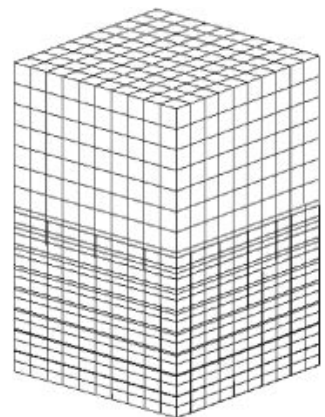

$48 \%$ reduction

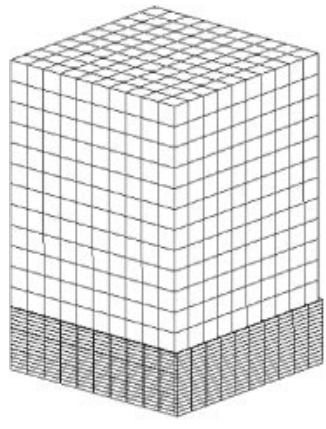

$80 \%$ reduction (a) SLIDING

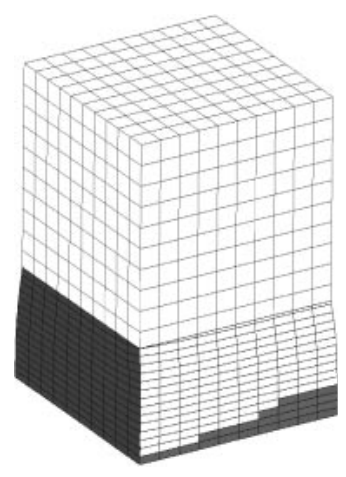

$65 \%$ reduction

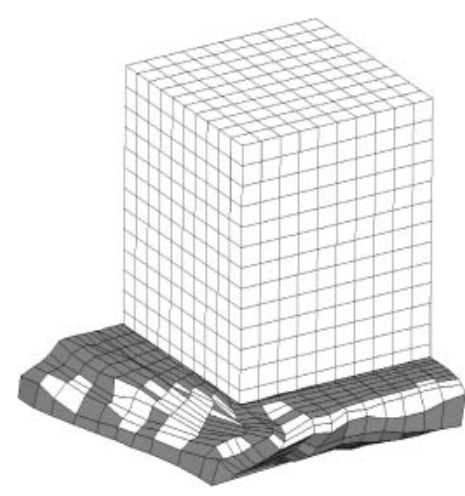

$85 \%$ reduction (b) STICKING

Fig. 4 Initial and deformed geometries predicted by FEM: (a) sliding $(\mu=0)$ and (b) sticking conditions.

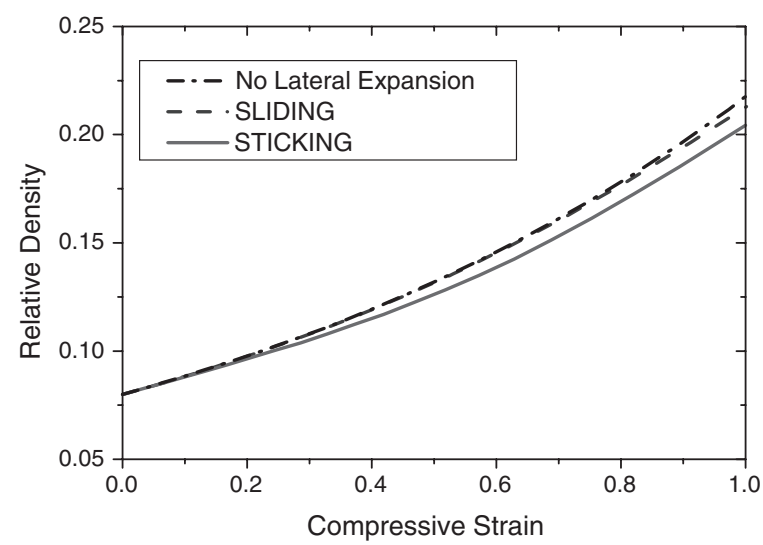

Fig. 5 Calculated average relative density as a function of compressive strain.

deformation is an important property in the engineering part design for service conditions. The volume contraction expressed by average relative density is shown in Fig. 5, where the average relative density was obtained by calculating the initial relative density multiplied by initial volume and divided by the deformed whole volume. It is not surprising that the relative density variation under the sliding condition is almost the same as that derived by the analytical solution assuming mass conservation and no lateral expansion. The relative density of the sticking condition is less than 


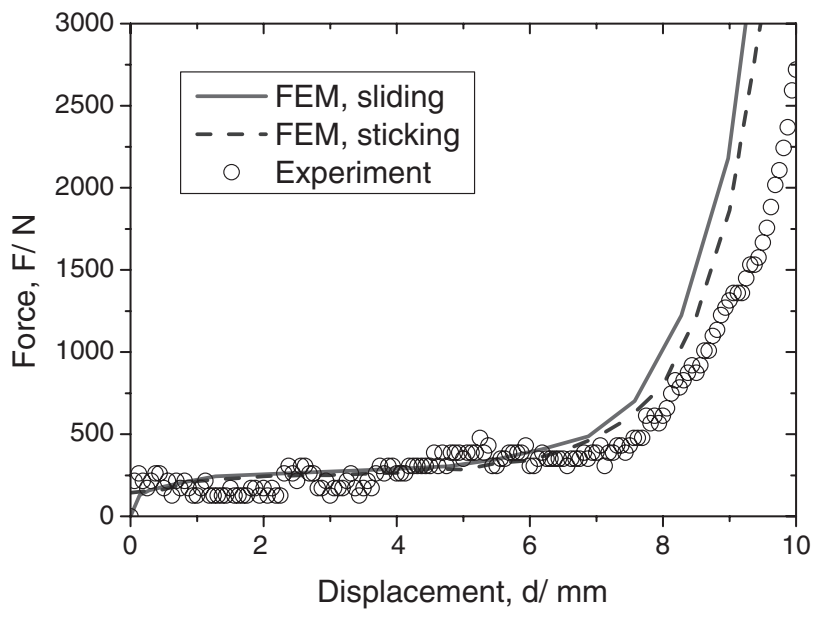

Fig. 6 Predicted and experimental load vs. displacement curves.

that of the sliding condition: $6 \%$ less at the compressive strain of 1.0. The less relative density can be understood by the deformed geometry and the resulting stress states; that is, the lateral constraint near the die contacting surface generates the more lateral outward flow in the central region (i.e. barreling) and the tensile stress on the central surface region. This tensile stress contributes less densification than the case of homogeneous deformation.

The predicted and experimental deforming forces in stages I and II in Fig. 6 represent a good agreement between experiment and simulations, where sliding and sticking results are not different in stages I and II. In stage III deformation, the sticking load is lower than the sliding load as a result of the development of local tensile stress and less densification due to the barreling effect. The experimental load is even lower than the sticking load of the simulation, meaning deformation is more heterogeneous possibly due to initial heterogeneous structure and imperfections. Considering the heterogeneities and experimental errors mostly from measuring dimensions, the finite element simulation using the crushable foam constitutive model is satisfactory and has a good potential for designing and developing practical engineering parts.

\section{Conclusions}

In this paper, deformation behavior of the $\mathrm{Al}-\mathrm{Mg}$ alloy foam material has been analyzed. Quasi-static compression of a cuboidal workpiece was modeled by the elasto-plastic finite element method based on the crushable foam constitutive model. The three deformation stages (elastic, plateau and densification) predicted by the finite element solutions were compared with the experimental data. The friction between the workpiece and die surfaces produced heterogeneous deformation and barreling, which played an important role in increasing tensile stress and decreasing densification rate and forming load. The finite element analysis associated with the crushable foam constitutive model has a good potential for designing and developing practical engineering parts.

\section{Acknowledgements}

This work was supported by Korea Research Foundation Grant (KRF-2003-005-D00012). The calculations were performed using the supercomputing resources of the Korea Institute of Science and Technology Information (KISTI).

\section{REFERENCES}

1) L. J. Gibson and M. F. Ashby: Cellular Solids: Structures and Properties, (Cambridge University Press, Cambridge, 1988).

2) F.-J. Ulm: Construction: in Cellular Materials in Encyclopedia of Materials: Science and Technology, Vol. 2, K. H. J. Buschow, R. W. Cahn, M. C. Flemings, B. Ilschner, E. J. Kramer and S. Mahajan ed. (Elsevier Science Ltd., Oxford, 2001) p. 1570.

3) J. Banhart: Prog. Mater. Sci. 46 (2001) 559-632.

4) M. J. Suk, S. I. Choi, J. S. Kim, Y. D. Kim and Y. S. Kwon: Metal. Mater. Inter. 9 (2003) 599-603.

5) K. Hashi, K. Ishikawa and K. Aoki: Metal. Mater. Inter. 7 (2001) 175179.

6) A. E. Markaki and T. W. Clyne: Acta Mater. 49 (2001) 1677-1686.

7) T. G. Nieh, K. Higashi and J. Wadsworth: Mater Sci. Eng. A283 (2000) 105-110.

8) P. Kenesei, Cs. Kadar, Zs. Rajkovits and J. Lendvai: Scr. Mater. 50 (2004) 295-300.

9) I. W. Hall, M. Guden and C.-J. Yu: Scr. Mater. 43 (2000) 515-521.

10) A. M. Hodge and D. Dunand: Metall. Mater. Trans. 34A (2003) 23532363.

11) H. S. Kim and D. N. Lee: Mater. Trans. 45 (2004) 1829-1832.

12) H. S. Kim, Y. Estrin, E. Gutmanas and C. K. Rhee: Mater. Sci. Eng. 307A (2001) 67-73.

13) A. G. Hanssen, M. Langseth and O. S. Hopperstad: Adv. Eng. Mater. 4 (2002) 771-776.

14) R. E. Miller: Int. J. Mech. Sci. 42 (2000) 729-754.

15) T. Naka and F. Yoshida: Metal Mater. Inter. 4 (1998) 464-466.

16) K. D. Woo, J. S. Lee and S. W. Kim: Metal Mater. Inter. 5 (1999) 363368.

17) M. F. Ashby, A. G. Evans, N. A. Fleck, L. J. Gibson, J. W. Hutchinson and H. N. G. Wadley: Metal Foam: A Design Guide, UK (2000) p. 13.

18) ABAQUS Inc.: ABAQUS Ver. 6.4 (2003).

19) D. N. Lee and H. S. Kim: Powder Metall. 35 (1992) 275-279.

20) H. S. Kim: Mater. Sci. Eng. A251 (1998) 100-105.

21) D.-S. Park, M.-W. Lee, H.-D. Kim and Y.-G. Jung: Met. Mater. Inter. 10 (2004) 393-398.

22) Y.-S. Kim and A. Needleman: Met. Mater. Inter. 9 (2003) 405-412.

23) J. Banhart and J. Baumeister: J. Mater. Sci. 33 (1998) 1431-1440.

24) H. Kanahashi, T. Mukai, Y. Yamada, K. Shimojima, M. Mabuchi, T. G. Nieh and K. Higashi: Mater. Sci. Eng. A280 (2000) 349-353. 